
About IJMA [last updated July, $\left.1^{\text {st }}, 2021\right]$

$\checkmark$ International Journal of Medical Arts is the Official Journal of the Damietta Faculty of Medicine, AlAzhar University, Egypt

$\checkmark$ It is an International, Open Access, Double-blind, Peer-reviewed Journal

$\checkmark$ Published four times a year

$\checkmark$ The First Issue was published in July 2019

$\checkmark$ Published under the following license: Creative Commons Attribution-ShareAlike 4.0 International Public License (CC BY-SA 4.0). It had updated from the Creative Commons license [CC BY] in volume 2, Issue 4, October 2020 About IJMA

$\checkmark$ The Egyptian Knowledge Bank hosts the web site of IJMA

$\checkmark$ The Egyptian Knowledge Bank supports IJMA

$\checkmark$ IJMA follows the regulations of the International Committee of Medical Journal Editors

$\checkmark$ IJMA is indexed in the "Directory of Open Access Journals" [15 January 2021].

$\checkmark$ IJMA is indexed in JGate [29-6-2021]

$\checkmark$ IJMA is a member of the International Society of Managing and Technical Editors

$\checkmark$ Listed in "Index Copernicus", "Publons", "Academic resource index [ResearchBib]", "Electronics journal library", "Eurasian Scientific Journal Index", and "Citefactor"

$\checkmark$ IJMA introduced to the search engine [BASE] through DOAJ
Click image to reach the page

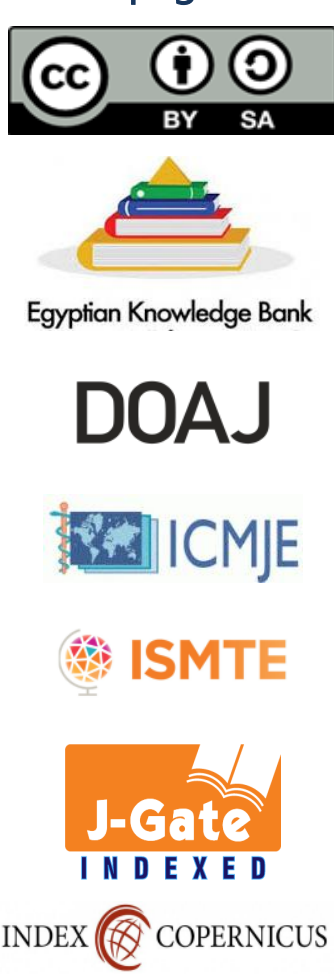

publons

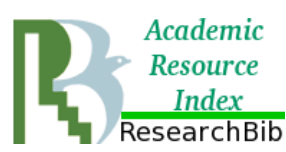

\section{EZ3 \\ .}

ESJII

CiteFactor

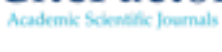

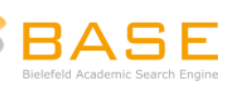




Available online at Journal Website
https://ijma.journals.ekb.eg/
Main subject [Basic Sciences; Microbiology]

Original Article

\title{
Detection of Biocides Resistance Genes among Clinical Isolates of Both Methicillin-Resistant and Methicillin-Sensitive Strains of Staphylococcus Aureus
}

\author{
Walid Sabry [1], Mohammed Yosry Salah Al-Dakhakhni [2], Ibrahim Mohammed Al-hoseiny [2], Raafat Abd Rabbo \\ Abdel Tawab [1].
}

${ }^{1}$ Department of Microbiology and Immunology, Damietta faculty of Medicine, Al-Azhar university, Egypt.

2 Department Medical Microbiology and Immunology, Faculty of Medicine, Al-Azhar University, Egypt.

Corresponding author: Walid Sabry

Email:walid.sabry@domazhermedicine.edu.eg

Submission date: February 08, 2021; Revision date: June 08, 2021; Acceptance date: June 11, 2021

BACKGROUND: Biocides compounds are widely used in infection control programs, including hand washing and skin decolonization before invasive procedures. However, its overuse leads to the emergence of Staphylococcus aureus with decreased biocides susceptibility, which became a problem in hospitals in different parts of the world.

AIM OF THE WORK: The present work aims to study the susceptibility of methicillin-resistant isolates of staphylococci aureus, obtained from different clinical samples to commonly used biocides, determines the prevalence of the biocide resistance genes, qac $A / B$, and qacC, among these isolates.

METHODS: Biocide's resistance genes [qacA/B, qacC] in Both Methicillin-Resistant and Methicillin-Sensitive Strains of Staphylococcus aureus were detected by PCR method. The minimum inhibitory concentrations [MICs] of Biocide agents were determined by broth dilution method, whereas antibiotic susceptibility was analyzed by disk diffusion and MICs methods according to Clinical and Laboratory Standards Institute [CLSI] criteria.

RESULTS: The frequency of Biocides resistance genes [qacA/B, qacC] was found to be high in our clinical staphylococci isolates. The qacA/B gene was detected in [63.3\%] of MRSA isolates compared with only [23.3\%] of MSSA isolates. Simultaneously, the qacC gene was detected in [63.3\%] of MRSA isolates compared to only [13.3\%] of MSSA isolates., with the significant difference between both groups $[\mathrm{P}<0.05]$. We also found an association between the presence of antiseptic resistance genes qacA/B, and increased MIC values to both savlon, hypochlorite, and Phenol $[P<0.05]$. We also showed that an association between the presence of antiseptic resistance genes qacC and the MICs to both savlon, hypochlorite, and Phenol $[P<0.05]$.

CONCLUSION: Our study detects biocide resistance genes [qacA/B, qacC] in Staphylococcus aureus isolates in Egypt; more studies are needed to correct the biocide uses and to support infection control programs to avoid the development of new resistance mechanisms.

Keywords: Resistance; Biocides; Staphylococcus aureus; Methicillin-Resistant; Biocides' resistance genes.

This is an open-access article registered under the Creative Commons, ShareAlike 4.0 International license [CC BY-SA 4.0] [https://creativecommons.org/licenses/by-sa/4.0/legalcode.

Citation: Sabry W, Al-Dakhakhni MYS, Al-hoseinyIM, Tawab A. Detection of Biocides Resistance Genes among Clinical Isolates of Both MethicillinResistant and Methicillin-Sensitive Strains of Staphylococcus Aureus. IJMA 2021;3(3): 1602 -1610. [DOI: 10.21608/JMMA.2021.62186.1260].

${ }^{*}$ Main subject and any subcategories have been classified according to the research topic. 


\section{INTRODUCTION}

Methicillin-resistant Staphylococcus aureus [MRSA] is an important nosocomial pathogen infecting individual in hospitals worldwide [1]. Many studies detect the important role of staphylococci aureus in disease in immunecompromised patients and the increased prevalence of multidrug-resistant strain, the emergence and rise of antibiotic resistance among staphylococci aureus is a burden in health care facilities and communities [2]

Biocides are chemical agents applied to inactivate or destroy microorganisms in different sites. Large amounts of biocides are used within different sites, including the medical environment used for disinfection, antisepsis, and cleaning. A wide variety of biocidal agents are commonly used in hospitals and healthcare facilities to decontaminate surfaces, disinfect the hands of health care personnel, and treat hospital-acquired infections ${ }^{[3]}$.

The worldwide increase in antimicrobial resistance in bacterial, leading to increased mortality and morbidity in humans, highlights the importance of infection control practices and the key role of healthcare biocides. However, the widespread use of biocides in hospitals has led to the emergence of disinfectant resistant bacteria. Excessive use may also result in the appearance of cross-resistance between widely used biocides and antibiotics ${ }^{[4]}$.

Epidemiological data on antiseptic susceptibility and the Distribution of resistance genes are useful for nosocomial infection control. Multidrug efflux systems are possibly the most described resistance mechanism regarding acquired resistance to biocides. Antiseptic resistance genes, qacA/B, qacC have been identified in Staphylococcus species ${ }^{[5]}$.

The qacA/B, qacC are found mainly in staphylococci aureus clinical isolates and could be responsible for reduced susceptibility to certain antiseptic agents. The incidence rate of MRSA in our hospital has increased to more than $80 \%$ of clinical isolates, and the prevalence of methicillin-resistant coagulase-negative staphylococci is also rising ${ }^{[6]}$.

\section{AIM OF THE WORK}

The present work aims to study the susceptibility of methicillin-resistant isolates of staphylococci Aureus, obtained from different clinical samples, commonly used biocides [antiseptic and disinfectant agents] to determine the prevalence of the biocide resistance genes, qacA/B, and qacC among these isolates.

\section{PATIENTS AND METHODS}

The present study was conducted on 600 strains isolated from hospital admitted patients, healthcare workers [HCWs], and environmental samples from different Clinical Departments of Al-Azhar University Hospital, Damietta Faculty of Medicine. From which 60 isolates of methicillinresistant staphylococci Aureus [MRSA] as well as 60 isolates of methicillin-sensitive staphylococci Aureus [MSSA] were taken to study the susceptibility to commonly used biocides and to determine the prevalence of the biocide resistance genes, qacA/B, and qacC, among these isolates. This work was conducted at the Microbiology Department of Damietta Faculty of Medicine from March 2019 to Jul 2020.

\section{1- Data collection}

Special questionnaires were prepared, including sex, age, health status, work type, medical history of the patients. Other data were also recorded for each HCWs. The samples were obtained from admitted patient's samples, Healthcare workers [HCWs] and environmental samples. Then, each sample was undergone to the following:

\section{I- Isolation and identification of staphylococci aureus by the following:}

[A] Direct Smear: Gram-stained smears: Typical staphylococci appear as gram-positive cocci in clusters.

[B] Culture and confirming the result:

- by inoculating them on blood agar and mannitol salt agar plates, given rise to typical colonies after 18-24 hours at $37^{\circ} \mathrm{C}$ in aerobic incubation ${ }^{[7]}$.

- Colonies suspected to be staphylococci aureus were confirmed by gram-stain, catalase, slide and tube coagulase, and deoxyribonuclease [DNase] tests ${ }^{[7]}$

\section{II- Detection of Methicillin Resistance:}

A] Determination of the susceptibility to Oxacillin and vancomycin antibiotics by the disc diffusion and minimal inhibitory concentration [MIC] methods: according to the recommendations of the [CLSI,2010, CLSI 2020] ${ }^{[8]}$.

B] Detection of penicillin-binding protein 2a by the SlidexR MRSA detection: MRSA isolates were tested for the presence of [PBP2a] [product of the mecA gene] by the Slidex ${ }^{\circledR M R S A}$ agglutination test ${ }^{[9]}$. 
III-Detection of staphylococci Aureus Susceptibility of both [MRSA] and [MSSA] isolates to Biocides by the Broth Dilution Method. The procedure was done by the serial two-fold broth dilution method as described by Mazzola et al. [10]. Biocides tested in the present study were selected from the chemical agents used to disinfect patient care items and instruments in the hospital. They were glutaraldehyde or cidex [2.0\% w/v] alkalinized with sodium bicarbonate $[\mathrm{pH}=8.3]$ supplied by Johnson and Johnson's; Sodium hypochlorite [10\% w/v] [pH 7.0] supplied by Elnasr Chemical Co.; Chlorhexidine digluconate [0.3\%]-Cetrimide [3\%] mixture [savlon] supplied by Cairo Pharma; aqueous polyvinyl-pyrrolidone-iodine $\left[10 \% \mathrm{w} / \mathrm{v}, \mathrm{PVP}-\mathrm{I}_{2}\right]$ solution supplied by Nile Co.; and Phenol [10\% w/v] solution supplied by El-Nasr Chemical Co. The MIC for each biocide was identified as the minimum concentration at which there was no visible growth.

IV- Detection of Biocides Resistance Genes by Polymerase Chain Reaction [PCR]: The qacA/B, qacC, resistance genes were detected in all isolates [both MRSA and MSSA isolates showed biocides resistance] by multiplex PCR according to lgnak et al. [2017] [11].

VI-Statistical analysis: Data was tabulated and analyzed using the Statistical Package for Social Sciences [SPSS] software, version 27 (IBM® SPSS® Inc., Armonk, USA). Data were presented as frequencies, with [P values $<0.05$ ] considered statistically significant.

\section{RESULTS}

The present study showed that the incidences of staphylococci aureus strain isolated from different samples of the study patients, HCWs and environmental strains isolated were 350 out of 600 [58.3\%] among all isolated strains, 269 out of 400 strains [67.2\%] were isolated from patients and 48 out 100 strains [48\%] were isolated from HCWs. In comparison, 33 out of 100 [33\%] were isolated from environmental samples, and the incidence of staphylococci aureus in patients was 269 out of 400 [67.2\%], 53 out of 400 [13.2\%] was MRSA, and 217 out of 400 [54.2\%] of them was MSSA. The incidence of staphylococci aureus isolates in HCWs was 48 out of 100 [48\%], of them 7 out of 100 [7\%] was MRSA, and 41 out of 100 [41\%] of them was MSSA. The incidence of staphylococci aureus in environmental samples was 33 out of 100 [33\%], all of them. And there was no significant difference between the three different methods, disc diffusion, MIC, and PBP2a production in patients, $\mathrm{HCW}$, and environmental samples.

Comparison between MRSA and MSSA isolates regarding the MIC to the different studied biocides shows a highly significant difference between both MRSA and MSSA isolates as regards the MICs to betadine [PVP-I $\left.\right|_{2]}$, Savlon, hypochlorite, and Phenol $[P<0.05]$. However, no significant difference was found between both groups as regards the MICs of the glutaraldehyde [Cadex] [P>0.05] [Table 1]. The Comparison between MRSA and MSSA isolates as regards the detection of biocides resistance genes [qac $A / B$ and qacC] shows; the qacA/B gene was detected in 38 out of 60 [63.3\%] of MRSA compared with only 14 out of 60 [23.3\%] of MSSA isolates. And the qacC gene was detected in 38 out of $60[63.3 \%]$ of MRSA and only in 8 out of 60 [13.3\%] of MSSA isolates. There was a highly significant association between both $[P<0.05]$. By the results of the PCR test, all isolates were classified into 4 groups: Group 1: Included 18 isolates [were +ve to qacA/B only]. Group 2: Included 12 isolates [were +ve to qacC only]. Group 3: Included 56 isolates [were -ve to both qacA/B and qacC]. Group 4: Included 34 isolates [were +ve to both qacA/B and qacC].

The comparison between group1 [qacA/B +ve] and group 3[qacA/B -ve and qacC -ve] as regards the MIC to the different studied biocides shows, there was no significant difference between group1 [qacA/B +ve] and group 3 [qacA/B ve and qacC -ve] as regards the MIC to betadine $\left[P V P-I_{2}\right]$ and glutaraldehyde [Cidex] [P>0.05]. However, there was an association between both groups regarding the MICs to savlon, hypochlorite, and Phenol $[P<0.05]$ [Table 2]. The comparison between group2 [qacC +ve] and group 3 [qacA/B -ve and qacC -ve] as regards the MIC to the different studied biocides shows, there was no significant difference between group2 [qacC $+\mathrm{ve}$ ] and group 3 [qacA/B -ve and qacC -ve] as regards the MICs to betadine $\left[\mathrm{PVP}-\mathrm{I}_{2}\right]$ and glutaraldehyde [Cidex] $[\mathrm{P}>0.05]$. However, a highly significant difference between both groups was detected regarding the MICs to savlon, hypochlorite, and Phenol $[\mathrm{P}<0.05]$ [Table3]. The comparison between group 4 [qacA/B +ve, qacC +ve] and group 3 [qacA/B -ve and qacC -ve] as regards the MIC to the different studied biocides shows, there was no significant difference between group 4 [qacA/B +ve, qacC $+v e$ ] and group 3 [qacA/B-ve and qacC -ve] as regards the MIC to glutaraldehyde [Cidex] [P>0.05]. However, a highly significant difference between both groups was detected as regards the MICs to betadine [PVP-I $\left.\right|_{2}$, savlon, hypochlorite, and Phenol $[P<0.05]$ [Table 4]. Comparing group1 [qacA/B +ve] and group 2 [qacC +ve] regarding the MIC to the different studied biocides, shows there was no significant difference between group 1 [qac $A / B+v e$ ], and group 2 [qacC $+v e]$ as regards the MICs to betadine [PVP-I $\left.\right|_{2}$, hypochlorite, and phenol $[P>0.05]$. However, there was a significant difference between both groups regarding the MICs to savlon $[P<0.05]$. However, a significant difference between both groups was detected regarding the MICs to savlon $[P<0.05]$ [Table 5]. 
Sabry W, et al.

IJMA 2021; 3 [3] July-September: 1602-1610

Table [1]: Comparison between MRSA and MSSA isolates regarding the MIC to the different studied biocides.

\begin{tabular}{|c|l|c|c|c||}
\hline \hline \multirow{2}{*}{ BIOCIDES } & CONC & \multicolumn{2}{|c|}{ Sensitive isolates } & PVALUE \\
[SIG.]
\end{tabular}

Table [2]: Comparison between group1 [qacA/B +ve] and group 3[qacA/B -ve and qacC -ve] as regards the MIC to the different studied biocides.

\begin{tabular}{|c|c|c|c|c|}
\hline \multirow[t]{2}{*}{ Biocides. } & \multirow[t]{2}{*}{ Conc } & \multicolumn{2}{|c|}{ Sensitive isolates } & \multirow{2}{*}{$\begin{array}{c}\text { P-value } \\
\text { [Sig.] }\end{array}$} \\
\hline & & Group 3 [AB-ve/C-ve] & Group1 [AB+ve/C-ve] & \\
\hline \multirow{3}{*}{$\begin{array}{l}\text { Betadine } \\
{\left[P \vee P-I_{2}\right]}\end{array}$} & 0.625 & 2 & 0 & \multirow{3}{*}{$\begin{array}{l}>0.05 \\
{[N S]}\end{array}$} \\
\hline & 1.25 & 32 & 6 & \\
\hline & 2.5 & 22 & 12 & \\
\hline \multirow{3}{*}{ Cidex } & 0.250 & 2 & 0 & \multirow{3}{*}{$\begin{array}{l}>0.05 \\
{[\mathrm{NS}]}\end{array}$} \\
\hline & 0.500 & 52 & 18 & \\
\hline & 1.000 & 2 & 0 & \\
\hline \multirow{3}{*}{ Savlon } & 0.051 & 46 & 0 & \multirow{3}{*}{$\begin{array}{c}<0.05 \\
{[S]}\end{array}$} \\
\hline & 1.65 & 6 & 8 & \\
\hline & 3.30 & 4 & 10 & \\
\hline \multirow{4}{*}{ Hypochlorite } & 0.206 & 46 & 0 & \multirow{4}{*}{$\begin{array}{c}<0.05 \\
{[S]}\end{array}$} \\
\hline & 0.825 & 8 & 10 & \\
\hline & 1.65 & 0 & 4 & \\
\hline & 3.30 & 2 & 4 & \\
\hline \multirow{4}{*}{ Phenol } & 0.078 & 46 & 0 & \multirow{4}{*}{$\begin{array}{c}<0.05 \\
{[S]}\end{array}$} \\
\hline & 0.625 & 0 & 2 & \\
\hline & 1.25 & 0 & 12 & \\
\hline & 2.50 & 10 & 4 & \\
\hline
\end{tabular}

Table [3]: Comparison between group2 [qacC +ve] and group 3 [qacA/B -ve and qacC -ve] as regards the MIC to the different studied

\begin{tabular}{|c|c|c|c|c|}
\hline \multirow{2}{*}{ Biocides } & \multirow[t]{2}{*}{ Conc } & \multicolumn{2}{|c|}{ Sensitive isolates } & \multirow{2}{*}{$\begin{array}{l}\text { P-value } \\
\text { [Sig.] }\end{array}$} \\
\hline & & Group 3 [AB-ve/C-ve] & Group $2[\mathrm{AB}-\mathrm{ve} / \mathrm{C}+\mathrm{ve}]$ & \\
\hline \multirow{3}{*}{$\begin{array}{l}\text { Betadine } \\
{\left[\mathrm{PVP}-\mathrm{I}_{2}\right]}\end{array}$} & 0.625 & 2 & 0 & \multirow{3}{*}{$\begin{array}{c}>0.05 \\
{[\mathrm{NS}]}\end{array}$} \\
\hline & 1.25 & 32 & 2 & \\
\hline & 2.5 & 22 & 10 & \\
\hline \multirow[t]{3}{*}{ Cidex } & 0.250 & 2 & 0 & \multirow{3}{*}{$\begin{array}{c}>0.05 \\
{[\mathrm{NS}]}\end{array}$} \\
\hline & 0.500 & 52 & 12 & \\
\hline & 1.000 & 2 & 0 & \\
\hline \multirow[t]{3}{*}{ Savlon } & 0.051 & 46 & 0 & \multirow{3}{*}{$\begin{array}{l}<0.05 \\
{[\mathrm{HS}]}\end{array}$} \\
\hline & 1.65 & 6 & 12 & \\
\hline & 3.30 & 4 & 0 & \\
\hline \multirow[t]{4}{*}{ Hypochlorite } & 0.206 & 46 & 0 & \multirow{4}{*}{$\begin{array}{c}<0.05 \\
{[\mathrm{HS}]}\end{array}$} \\
\hline & 0.412 & 0 & 2 & \\
\hline & 0.825 & 8 & 10 & \\
\hline & 3.30 & 2 & 0 & \\
\hline \multirow[t]{4}{*}{ Phenol } & 0.078 & 46 & 0 & \multirow{4}{*}{$\begin{array}{l}<0.05 \\
{[\mathrm{HS}]}\end{array}$} \\
\hline & 0.625 & 0 & 6 & \\
\hline & 1.25 & 0 & 6 & \\
\hline & 2.50 & 10 & 0 & \\
\hline
\end{tabular}


Table [4]: Comparison between group 4 [qacA/B +ve, qacC +ve] and group 3 [qacA/B -ve and qacC -ve] as regards the MIC to the different studied biocides.

\begin{tabular}{|c|c|c|c|c|}
\hline \multirow[t]{2}{*}{ Biocides } & \multirow[t]{2}{*}{ Conc } & \multicolumn{2}{|c|}{ Sensitive isolates } & \multirow{2}{*}{$\begin{array}{c}\text { P-value } \\
\text { [Sig.] }\end{array}$} \\
\hline & & Group 3 [AB-ve/C-ve] & Group $4[\mathrm{AB}+\mathrm{ve} / \mathrm{C}+\mathrm{ve}]$ & \\
\hline \multirow[t]{3}{*}{ Betadine $\left[P V P-I_{2}\right]$} & 0.625 & 2 & 0 & \multirow{3}{*}{$\begin{array}{l}<0.05 \\
{[\mathrm{HS}]}\end{array}$} \\
\hline & 1.25 & 32 & 2 & \\
\hline & 2.5 & 22 & 32 & \\
\hline \multirow[t]{3}{*}{ Cidex } & 0.250 & 2 & 0 & \multirow{3}{*}{$\begin{array}{c}>0.05 \\
{[N S]}\end{array}$} \\
\hline & 0.500 & 54 & 34 & \\
\hline & 1.000 & 2 & 0 & \\
\hline \multirow[t]{3}{*}{ Savlon } & 0.051 & 46 & 0 & \multirow{3}{*}{$\begin{array}{c}<0.05 \\
{[\mathrm{HS}]}\end{array}$} \\
\hline & 1.65 & 6 & 16 & \\
\hline & 3.30 & 4 & 18 & \\
\hline \multirow[t]{4}{*}{ Hypochlorite } & 0.206 & 46 & 0 & \multirow{4}{*}{$\begin{array}{c}<0.05 \\
{[\mathrm{HS}]}\end{array}$} \\
\hline & 0.825 & 8 & 22 & \\
\hline & 1.65 & 0 & 4 & \\
\hline & 3.30 & 2 & 8 & \\
\hline \multirow[t]{5}{*}{ Phenol } & 0.078 & 46 & 0 & \multirow{5}{*}{$\begin{array}{c}<0.05 \\
{[\mathrm{HS}]}\end{array}$} \\
\hline & 0.312 & 0 & 2 & \\
\hline & 0.625 & 0 & 8 & \\
\hline & 1.25 & 0 & 18 & \\
\hline & 2.50 & 10 & 6 & \\
\hline
\end{tabular}

NS=non-significant; HS = highly significant

Table [5]: Comparison between group1 [qacA/B +ve] and group 2 [qacC +ve] as regards the MIC to the different studied biocides.

\begin{tabular}{|c|c|c|c|c|}
\hline \multirow[t]{2}{*}{ Biocides } & \multirow[t]{2}{*}{ Conc } & \multicolumn{2}{|c|}{ Sensitive isolates } & \multirow[t]{2}{*}{ P-value [sig.] } \\
\hline & & Group $1[\mathrm{Ve}+\mathrm{AB} / \mathrm{C}-\mathrm{ve}]$ & Group $2[\mathrm{Ve}-\mathrm{AB} / \mathrm{C}+\mathrm{ve}]$ & \\
\hline \multirow[t]{2}{*}{ betadine $\left[\mathrm{PVP}-\mathrm{I}_{2}\right]$} & 1.25 & 6 & 2 & \multirow{2}{*}{$\begin{array}{l}>0.05 \\
{[N S]}\end{array}$} \\
\hline & 2.5 & 12 & 10 & \\
\hline \multirow[t]{2}{*}{ Savlon } & 1.65 & 8 & 12 & \multirow{2}{*}{$\begin{array}{c}<0.05 \\
{[S]}\end{array}$} \\
\hline & 3.30 & 10 & 0 & \\
\hline \multirow[t]{4}{*}{ Hypochlorite } & 0.412 & 0 & 2 & \multirow{4}{*}{$\begin{array}{l}>0.05 \\
{[N S]}\end{array}$} \\
\hline & 0.825 & 10 & 10 & \\
\hline & 1.65 & 4 & 0 & \\
\hline & 3.30 & 4 & 0 & \\
\hline \multirow[t]{3}{*}{ Phenol } & 0.625 & 2 & 6 & \multirow{3}{*}{$\begin{array}{c}>0.05 \\
{[N S]}\end{array}$} \\
\hline & 1.25 & 12 & 6 & \\
\hline & 2.50 & 4 & 0 & \\
\hline
\end{tabular}

\section{DISCUSSION}

The present work aimed to study the susceptibility of methicillin-resistant Staphylococcus aureus, MRSA, and MSSA strains, obtained from different samples, to commonly used biocides and detect the presence of the biocide's resistance genes, qacA/B and qacC, among these isolates. The present study showed that all incidences of staphylococci aureus strain isolated from different samples of the study patients, HCWs and environmental strains isolated were 350 out of 600 [58.3\%] among all isolated strains, 269 out of 400 strains [67.2\%] were isolated from patients and 48 out 100 strains [48\%] were isolated from HCWs, while 33 out of 100 [33\%] were isolated from environmental samples. Our results demonstrated that a difference of the incidence of staphylococci aureus isolated from different samples of the study, being [67.2\%] as regard to patients, and [48\%] as regard to $\mathrm{HCWs}$, and [33\%] as regard to environmental samples, with statistically significant difference. In the study conducted between August 2011 and June 2012, at Al- Kadhamia teaching Hospital and AlNuman hospital, Baghdad, Iraq ${ }^{[12]}$. One hundred and six staphylococci aureus were isolated from patients and Healthcare workers. The incidence of staphylococci aureus in this study was $42.4 \%$. which was lower than our results [58.3\%]. Other study conducted at AlZahraa University Hospital included admitted 300 female patients to identify the causative organism[s] responsible for surgical site infection following Cesarean section. The most common organism isolated was staphylococci aureus $31 / 300$ [10.3\%] [13], which was lower than our results [58.3\%].

In our study, the staphylococci aureus isolated strains were 350 out of 600 [58.3\%]. This result compared to those obtained by ${ }^{[14]}$ with staphylococci aureus percentages of $31.27 \%$. The high rates of staphylococci aureus contamination in our study are likely to reflect both poor hand hygiene practices and compromised hygienic measures and increased duration of hospital stay.

As regard to patients' group, MRSA were isolated 53 out of 400 [13.2\%] compared 216 out of 400 [54\%] of MSSA. While HCWs group MRSA strains were isolated was 7 out of 100 [7\%] compared to 41 out of 100 [41\%] of MSSA, and environmental group, all environmental isolates 33 out of 
100 [33\%] were MSSA. There was a significant difference between both MRSA and MSSA isolates in the three groups. In the study conducted in north Jordan, the incidence of staphylococci aureus in patients were [19.8\%], [5.8\%] were found to be MRSA ${ }^{[15]}$. There is a difference in the incidence of both staphylococci aureus [19.8\%], and MRSA [5.8\%] between this study and our study. In our study a higher incidence of both staphylococci aureus [67.2\%], and MRSA [13.2\%], with statistically significant difference. In another study, conducted at Fahd hospital, Al-Madinah AlMunawarah, KSA on patients, [HCWs] and environment, samples isolated from 117 patients, $25 \mathrm{HCWs}$ and 12 environmental sites ${ }^{[16]}$. The rate of MRSA was $5.98 \%$ [7/117] in patients. which was lower than our results [13.2\%.], the rate of MRSA was $36 \%$ [9/25] in HCWs which was higher than our results [7\%.]. Two MRSA strains were isolated from the 12 environmental samples compared to no environmental MRSA in our results. In another trial was undertaken between August 2011 and June 2012, at AlKadhamia teaching Hospital and Al-Numan hospital, Baghdad, Iraq [11]. One hundred and six staphylococci aureus were isolated from patients and Healthcare workers. The incidence of staphylococci aureus in this study was $42.4 \%$. which was lower than our results [58.3\%]. The rate of MRSA in patients was $97 \%$ which was higher than our results [13.2\%.], the rate of MRSA in $\mathrm{HCWs}$ was $82 \% \%$ which was higher than our results [7\%.], which confirm the higher incidence rate of MRSA in both patients and HCWs in this study thank our study.

In another study was conducted at $\mathrm{MNH}$ in Dar es Salaam, Tanzania, between May and June 2018, on environmental samples, the results of this study showed that staphylococci aureus incidence was [20\%] from environmental samples, which was lower than our results [33\%], these results including $19.5 \%$ for MRSA, compared with our results $[0 \%]$ for MRSA ${ }^{[17]}$.

Another study was conducted at Al Zahraa University Hospital on 300 females admitted patients to identify the most causative organism[s] responsible for surgical site infection after Cesarean section. The most common organism isolated was staphylococci aureus [10.3\%] [13], which was lower than our results [58.3\%].

Staphylococci aureus isolates were then identified according to sensitivity to oxacillin, revealing 21 isolates were MRSA 21/300 [7\%] among isolates of staphylococci aureus strains. The rate of MRSA was $7 \%$ in patients, which was lower than our results [13.2\%.], and from [HCWs] \& environment collected swabs, [26\%] were positive on culture, 18 isolates [5.6\%] were obtained from HCWs. 65 isolates were isolated from environmental samples [20\%].
The most common organism isolated from both sources [clinical \& environmental specimens] was [MRSA] isolates $[5.6 \%]^{[13]}$, which was lower than our results in both [HCWs] 7out of $100[7 \%]$ and \& environmental samples [0\%].

Other study conducted at Al-Azhar University Hospital, Damietta branch. The results obtained in Egyptian study by Alaa Eldeen et al. ${ }^{[18]}$ revealed MRSA nasal carriage rate of [2.3\%] among medical residents in Gynecology department which is lower than our study results about MRSA isolates in [HCWs] [7\%]. Our study result is higher than the study result conducted by Siegel et al. ${ }^{[19]}$, who reported an MRSA colonization rate of $2.1 \%$ in the nasal cavity and axilla of HCWs.

The high rates of MRSA contamination in [HCWs] are likely to be a source of infection and transmission of pathogens within the hospital and remains a hazard for hospitalized patients. Also, cross-transmission of microorganisms from patients and the environment may have an important role.

The high MRSA contamination rates reflect both poor hand hygiene practices and compromised hygienic measures. Increased duration of stay in hospitals. The difference in MRSA rates in different studies might be due to the difference in locations and periods of the studies, the difference in hygienic conditions maintained in different hospitals, the hospital's healthcare facilities, and the implementation of the program for infection control, and rational use of antibiotics.

As regarding the MIC of both [MRSA and MSSA] isolates to the different studied biocides, both MRSA and MSSA isolates were effectively inhibited by the user's concentrations of betadine [PVP-I $\left.{ }_{2}\right][2.5 \%]$, glutaraldehyde [0.5\%], Savlon [0.825\%], hypochlorite [3.3\%] and Phenol [1.25\%]. However, the MRSA isolates had statistically higher MICs to betadine [PVP-| $\left.\left.\right|_{2}\right][P<0.05]$ as compared to MSSA isolates. This finding goes in agreement with Zhang et al. [20], who also found that MRSA isolates had increased MIC to povidone-iodine [betadine] as compared to the MSSA isolates. By contrast, Bamber and Neal [21], reported no differences between the sensitivity of MRSA and MSSA to betadine. Also, a highly significant difference was observed between both [MRSA and MSSA] regarding the MICs to Savlon $[P<0.05]$. This finding goes in accordance with the previous finding of Irizarry et al. [22], who also found the increased value of MICs of MRSA strains for some biocides, including cetrimide and chlorhexidine. Also, Wootton et al. ${ }^{[23]}$ observed that the MICs for QACs for MRSA were 1.5-3-fold higher than those for MSSA. However, Al-Masaudi et al. ${ }^{[24]}$ found that there was no 
important difference between MRSA and MSSA regarding their susceptibility to savlon.

Unlike the results by Al-Masaudi et al. ${ }^{[24]}$ who found that both MRSA and MSSA are equally sensitive to phenols, a highly significant difference in the present study, between the MRSA and MSSA isolates regarding the MICs to Phenol $[P<0.05]$ was observed.

Regarding hypochlorite sensitivity in the present work, the MRSA isolates had significantly higher MICs than the MSSA isolates $[P<0.05]$. The MRSA isolates MIC value exceeded the user-defined concentrations [MIC $=0.825]$. This finding supports the finding of Mitchell et al. [25], who reported an increased MIC for MRSA than MSSA isolates to hypochlorite.

In our study, no significant difference was reported between both MRSA and MSSA isolates regarding the MICs of the glutaraldehyde and glutaraldehyde concentration as low as $0.5 \%$ effectively inhibited all isolates. This finding is in accordance with the findings of Reynaldo et al. [26] who reported that MRSA isolates are sensitive to $2 \%$ glutaraldehyde as MSSA. Again, Kheiralla et al. [27] noted, the highest concentration of glutaraldehyde [2\%] was the most concentration effective against the MRSA isolates. Also, the authors noted that the $0.5 \%$ concentration was more effective on the MRSA isolates than the concentration of $1 \%$.

The studied MRSA isolates were sensitive to the most used disinfectants/ antiseptics at the user's defined concentrations, and there was an important difference between the MICs of MRSA isolates as compared to the MSSA isolates. This is important as it may mean that certain isolates have an ability to survive at lower concentrations of biocides and that the use of biocides may act as a selective pressure to allow these isolates to predominate.

As regards the presence of biocides resistance genes [qacA/B and qacC] in both MRSA and MSSA isolates. In the current work, the qacA/B gene was present in $43.3 \%$ of the studied staphylococci aureus isolates. There was a significant difference between the MRSA isolates and the MSSA isolates regarding the presence of the qacA/B gene since the gene was presented in $63.3 \%$ of MRSA isolates and in $23.3 \%$ of MSSA isolates $[P<0.05]$. This goes in agreement with the study of Mayer et al. [28], who detected the qacA/B genes in $42 \%$ of their studied staphylococci aureus strains were isolated between 1997 and 1999 from 24 different European university hospitals. The authors noticed significantly more MRSA than MSSA isolates possessed the qacA/B genes $[63 \%$ versus $12 \%$, respectively]. Also, Taheri et al. ${ }^{[29]}$ detected the qacA/B gene in $76.46 \%$ of their studied staphylococci aureus strains isolates. The authors noticed that significantly more MRSA than MSSA isolates possessed the qacA/B genes [63.3\% versus $25.9 \%$, respectively].

Also, Noguchi et al. [30] detected the qacA/B gene in $41.6 \%$ of MRSA strains isolated from different Asian countries between 1998 and 1999. Later, Sekiguchi et al. [31] reported a higher detection rate of the qacA/B gene [52.3\%] among their studied MRSA isolates from a Tokyo hospital. Furthermore, Noguchi et al. ${ }^{[30]}$ detected the [qacA/B] gene in $45.9 \%$ of MRSA isolates cultured from patients. A much higher rate was reported by Miyazaki et al. [32], who found the [qacA/B] gene in $80 \%$ of MRSA strains isolated in Brazil. Also, Conceicao et al. ${ }^{[33]}$ detected the qacA/B gene among $40.5 \%$ of MRSA strains isolates. Also, Wang et al. [34] detected the [qacA/B] gene among $61.1 \%$ of MRSA strains isolated in China.

On the other side, Vali et al. [35] detected the [qacA/B] gene in only $10 \%$ of their studied isolated MRSA from hospitals in Edinburgh. Also, Ignak et al. [11] detected the [qacA/B] gene among $10.3 \%$ of MRSA isolates.

In the present work, the qacC gene was presented in $38.3 \%$ of the studied staphylococci aureus isolates. The gene was presented in $63.3 \%$ of the MRSA strains isolates and in $13.3 \%$ of the MSSA isolates $[P<0.05]$. These results are much higher than those reported by Alam et al. ${ }^{[36]}$ and Noguchi et al. [29], who reported the qacC gene in $3.3 \%$ and $2.5 \%$ only of their studied MRSA isolates, respectively. Also, our results are much higher than those reported by Conceicao et al. [33], and Taheri et al. [29], who detected the qacC gene in $3.7 \%$, and $6.7 \%$ only of their studied MRSA isolates, respectively. However, Vali et al. ${ }^{[35]}$ noted that the qacC gene was more spread [44.2\%] than the [qacA/B] gene [10\%] among their studied MRSA strains isolated from hospitals in Edinburgh. Also, Ignak et al. [11] noted that the qacC gene was more spread [13.8\%] than the [qacA/B] gene [10.3\%] among their studied MRSA strains isolated from hospitals. The high spreading of these genes is likely to be due to selective pressure by the various disinfection agents used in hospitals. Moreover, it is noted that more MRSA than MSSA isolates was positive for the [qacA/B] gene. This may be due to [the location of the qacA/B genes on multi-resistance plasmids like the pSK qacA] and the $\beta$ lactamase/heavy metal resistance families], such as pSK23. These plasmids harbor a few determinants responsible for resistance to a range of antimicrobial agents. This finding plays an important role in qacA/B genes in biocide resistance.

In the present trial, a highly significant difference was 
found between group 1 [qacA/B +ve] and group 3 [qac $A / B$ and gacC -ve] regarding the MICs to savlon, hypochlorite, and Phenol. Yet, no significant difference was observed between both groups regarding the susceptibility to betadine $\left[\mathrm{PVP}-\mathrm{I}_{2}\right]$ and glutaraldehyde. Behr et al. ${ }^{[37]}$ also observed a significant difference between these isolates that carry qacA/B and those that do not carry qacA/B regarding MICs of QACs and chlorohexidine.

In our study, a highly significant difference was found between group 2 [qacC +ve] and group 3 [qacA/B -ve and qacC -ve] regarding the MICs to savlon, hypochlorite, and Phenol. Yet, no significant difference was detected between both groups regarding to the susceptibility to betadine [PVP$\mathrm{I}_{2}$ ] and glutaraldehyde. Similarly, Mycock et al. ${ }^{[35]}$ reported the increased MICs for isolates carrying the qacC gene of biocides, including chlorhexidine, cetrimide, benzalkonium chloride, and hypochlorite. Also, Sekiguchi et al. [31] reported a significant difference between the isolated strains that carry the qacC gene and those that do not carry the qacC gene regarding the MICs of QACs.

In the current study, a highly significant difference was found between group 4 [qacA/B +ve, qacC $+v e]$ and group 3 [qacA/B-ve and qacC -ve] regarding the MICs to betadine [PVP-I $I_{2}$, savlon, hypochlorite and Phenol. In agreement with Al-Masaudi et al. [24] who reported increased MICs against plasmid-carrying MRSA strains for biocides including chlorhexidine, cetrimide, benzalkonium chloride, hypochlorite, and betadine. In addition, a significance difference was found between group1 [qacA/B +ve] and group 2 [qacC $+v e]$ regarding the MICs to savlon with the qaCA/B +ve group being more resistant than the qacC +ve group to savlon. No significant difference was detected between both groups regarding their susceptibility to betadine [PVP- $\left.\mathrm{I}_{2}\right]$, glutaraldehyde, hypochlorite and phenol. This finding goes in accordance with Russell et al. [39] who reported that the qacA/B gene specifies resistance to chlorhexidine and QACs whereas the qacC gene carry resistance to some QACs as previously reported by Leelaporn et al. ${ }^{[40]}$

From the current study we concluded that although the studied MRSA isolates strains were susceptible to most of the commonly used disinfectants/antiseptics at the user's defined concentrations, there was a significant difference between the MICs of MRSA isolates strains as compared to the MSSA isolates. This is of importance as it may mean that certain isolates will have an ability to survive at lower concentrations of biocides, the use of biocides act as a selective pressure to allow these isolates to predominate. Thus, we recommend eradicating MRSA colonization in both patients and HCWs to prevent its spread to the community. Further studies on larger number of isolates to confirm our findings. Other studies on other multi-drug resistant organisms particularly gram-negative organisms are also recommended.

Financial and Non-financial Relationships and Activities of Interest

None

\section{REFERENCES}

1.Machado TS, Pinheiro FR, Andre LSP, Pereira RFA, Correa RF, de Mello GC, et al. Virulence Factors Found in Nasal Colonization and Infection of Methicillin-Resistant Staphylococcus aureus [MRSA] Isolates and Their Ability to Form a Biofilm. Toxins. 2021; 13[1]:14. [DOI:10.3390/toxins13010014].

2.Mosselhy DA, Assad M, Sironen T, Elbahri M. Nanotheranostics: A Possible Solution for Drug- Resistant Staphylococcus aureus and their Biofilms? Nanomaterials. 2021; 11[1]:82. [DOI: 10.3390/ nano11010082].

3. Elekhnawy E, Sonbol F, Abdelaziz A, Elbanna T. Potential impact of biocide adaptation on selection of antibiotic resistance in bacterial isolates. Futur J Pharm Sci. 2020; 6: 97. [DOI: 10.1186/s43094-02000119-w].

4. Herault HSS. Impact of commonly used antimicrobial biocides on resistance and cross-resistance in carbapenemase-producing Enterobacteriaceae. PhD Thesis, Cardiff University, 2020, available at: https://ethos.bl.uk/OrderDetails.do?uin=uk.bl.ethos.805727. Last accessed March 2021.

5.Ye JZ, Yu X, Li XS, Sun Y, Li MM, Zhang W, et al. [Antimicrobial resistance characteristics of and disinfectant resistant gene distribution in Staphylococcus aureus isolates from male urogenital tract infection]. Zhonghua Nan Ke Xue, 2014 Jul;20[7]:630-636. [English Abstrat].

6. Rezazadeh M, Yousefi Mashouf R, Sarmadyan H, Ghaznavi-Rad E. Antibiotic profile of methicillin-resistant Staphylococcus aureus with multiple-drug resistances isolated from nosocomial infections in Valiasr Hospital of Arak. Arak Medic J. 2013;16[2]:29-37.

7. Geo. FB, Karen CC, Janet SB, Stephen AM, Jawetz M. Adelberg's Medical Microbiology 2019, twenty-fifth edition. ISBN 978-1-26001202-6; McGraw-Hill Education; pp 250-260.

8.Clinical and Laboratory Standards Institute [CLSI]. Performance standards for antimicrobial susceptibility testing. $18^{\text {th }}$ information supplement; M100-S18 [2020]. Available at: https://clsi.org/ standards/ products/microbiology/documents/m100/; Last accessed March 2021.

9. Datta P, Gulati N, Singla N, Rani Vasdeva H, Bala K, Chander J, Gupta $V$. Evaluation of various methods for the detection of meticillinresistant Staphylococcus aureus strains and susceptibility patterns. J Med Microbiol. 2011 Nov;60[Pt 11]:1613-1616. [DOI: 10.1099/jmm. 0.032219-0].

10. Mazzola PG, Jozala AF, Novaes L, Moreial P, Vessoni TCP. Minimal inhibitory concentration [MIC] determination of disinfectant and/or sterilizing agents. Brazilian J Pharmaceut. Sci 2009: 45: 241-248. [DOI: 10.1590/S1984-82502009000200008].

11. Ignak $S$, Nakipoglu $Y$, Gurler B. Frequency of antiseptic resistance genes in clinical staphycocci and enterococci isolates in Turkey. Antimicrob Resist Infect Control. 2017; 6:88. [DOI: 10.1186/s13756017-0244-6].

12. Al-Dahbi AM, Al-Mathkhury HJ. Distribution of Methicillin Resistant Staphylococcus aureus in Iraqi patients and Healthcare Workers. Iraqi J Sci. 2015: 54 [2]: 293-300. 
13. El-Nasser AM, El Salakawy AH, Mira AA, Ibrahim DF, El-Sharaky HF. Epidemiological Typing of Methicillin Resistant Staphylococcus Aureus Isolated from Surgical Site Infection Following Caesarean Section in an Egyptian University Hospital. Egy J Hosp Med. 2019; 77 [5]: 5534-5541. [DOI: 10.21608/ejhm.2019.60861].

14. Kathryn CK, Maine R, Trelles M. Cesarean Section Surgical Site Infections in Sub-Saharan Africa: A Multi-Country Study from Medecins Sans Frontieres. World J Surg. 2015: 39: 350-355. [DOI: 10.1007/s00268-014-2840-4].

15. Resić H, Corić A, Dedeić-Ljubović A, Hukić M, Avdić E, Kukavica N. [Prevalence of MRSA infections in patients on hemodialysis]. Med Pregl. 2007;60 Suppl 2:97-100. Serbian. [PMID: 18928170].

16. El-Ageery SM, Abo-Shadi MA, Elgendy AM, Alghaithy AA, Kandeel AY. The Role of Health Care Workers and Environment on Transmission of Methicillin-Resistant Staphylococcus aureus among Patients in a Medical Intensive Care Unit in a Saudi Hospital. J Pure Applied Microbiol. 2011; 5[1]: 7 pages.

17. Nkuwi EJ, Kabanangi $F$, Joachim A, Rugarabamu S, Majigo M. Methicillin-resistant Staphylococcus aureus contamination and distribution in patient's care environment at Muhimbili National Hospital, Dar es Salaam-Tanzania. BMC Res Notes. 2018 Jul 17;11[1]:484. [DOI: 10.1186/s13104-018-3602-4].

18. Alaa Eldeen MH, Ali I, Khaled E, Mohammed AA, Hany A, Ahmed AE, Fathia ME. Nasal colonization of methicillin-resistant Staphylococcus aureus among medical residents in Al-Azhar University Hospital, Damietta branch. AZMJ 2018: 16: 87- 95. [DOI: 10.4103/1687-1693.244149].

19. Siegel GW, Patel NN, Milshteyn MA, Buzas D, Lombardo DJ, Morawa LG. Cost Analysis and Surgical Site Infection Rates in Total Knee Arthroplasty Comparing Traditional vs. Single-Use Instrumentation. J Arthroplasty. 2015 Dec;30[12]:2271-4. [DOI: 10.1016/j.arth.2015.05.037].

20. Zhang YH, Liu XY, Zhu LL, Yu YZ. [Study on the resistance of methicillin-resistant staphylococcus aureus to iodophor and chlorhexidine]. Zhonghua Liu Xing Bing Xue Za Zhi. 2004 Mar;25[3]:248-50. Chinese. [PMID: 15200941].

21. Bamber Al, Neal TJ. An assessment of triclosan susceptibility in methicillin-resistant and methicillin-sensitive Staphylococcus aureus. J Hosp Infect. 1999 Feb; 41[2]:107-9. [DOI: 10.1016/s01956701[99]90047-6].

22. Irizarry L, Merlin T, Rupp J, Griffith J. Reduced susceptibility of methicillin-resistant Staphylococcus aureus to acetylpyridinium chloride and chlorhexidine. Chemotherapy. 1996 Jul-Aug; 42[4]:24852. [DOI: $10.1159 / 000239451]$.

23. Wootton M, Walsh TR, Davies EM, Howe RA. Evaluation of the effectiveness of common hospital hand disinfectants against methicillin-resistant Staphylococcus aureus, glycopeptide-intermediate $S$. aureus, and heterogeneous glycopeptide-intermediate $S$. aureus. Infect Control Hosp Epidemiol. 2009 Mar; 30 [3]: 226-32. [DOI: 10.1086/595691].

24. Al-Masaudi SB, Day MJ, Russell AD. Sensitivity of methicillinresistant Staphylococcus aureus strains to some antibiotics, antiseptics, and disinfectants. J Appl Bacteriol. 1988 Oct;65[4]:32937. [DOI: 10.1111/j.1365-2672. 1988.tb01899. x].

25. Mitchell BA, Brown MH, Skurray RA. QacA multidrug efflux pump from Staphylococcus aureus: comparative analysis of resistance to diamidines, biguanidines, and guanylhydrazones. Antimicrob Agents Chemother. 1998 Feb;42[2]:475-7. [DOI: 10.1128/AAC.42.2.475].

26. Reynaldo MB, Flores MB, Viegas Caetano JA, Magariños Mdel C. [Efficacy of biocides against hospital isolates of Staphylococcus sensitive and resistant to methicillin, in the province of Buenos Aires, Argentina]. Rev Panam Salud Publica. 2004 Sep;16[3]:187-92. Spanish. [DOI: 10.1590/s1020-49892004000900005].

27. Kheiralla Z, Abdelnasser M, Rushdy A, Osman AS. The efficacy of combination of disinfectants or antiseptics in overcoming methicillinresistant Staphylococcus aureus. J Pure Applied Microbio 2014; 8 [2]:1611-1618.

28. Mayer S, Boos M, Beyer A, Fluit AC, Schmitz FJ. Distribution of the antiseptic resistance genes qacA, qacB and qacC in 497 methicillinresistant and -susceptible European isolates of Staphylococcus aureus. J Antimicrob Chemother. 2001 Jun; 47[6]:896-7. [DOI: 10.1093/jac/47. 6.896].

29. Taheri N, Ardebili A, Amouzandeh-Nobaveh A, Ghaznavi-Rad E. Frequency of Antiseptic Resistance Among Staphylococcus aureus and Coagulase-Negative Staphylococci Isolated from a University Hospital in Central Iran. Oman Med J. 2016 Nov;31[6]:426-432. [DOI: 10.5001/omj.2016.86]

30. Noguchi N, Suwa J, Narui K, Sasatsu M, Ito T, Hiramatsu K, Song $\mathrm{JH}$. Susceptibilities to antiseptic agents and distribution of antisepticresistance genes qacA/B and SMR of methicillin-resistant Staphylococcus aureus isolated in Asia during 1998 and 1999. J Med Microbiol. 2005 Jun; 54 [Pt 6]: 557-565. [DOI: 10.1099/jmm.0.459020].

31. Sekiguchi J, Hama T, Fujino T, Araake M, Irie A, Saruta K, et al. Detection of the antiseptic- and disinfectant-resistance genes qacA, qacB, and qacC in methicillin-resistant Staphylococcus aureus isolated in a Tokyo hospital. Jpn J Infect Dis. 2004 Dec; 57[6]:28891. [PMID: 1562396].

32. Miyazaki NH, Abreu AO, Marin VA, Rezende CA, Moraes MT, Villas Bôas $\mathrm{MH}$. The presence of qacA/B gene in Brazilian methicillinresistant Staphylococcus aureus. Mem Inst Oswaldo Cruz. 2007 Jun; 102 [4]:539-40. [DOI: 10.1590/s0074-02762007000400018].

33. Conceição T, Coelho C, de Lencastre H, Aires-de-Sousa M. High Prevalence of Biocide Resistance Determinants in Staphylococcus aureus Isolates from Three African Countries. Antimicrob Agents Chemother. 2015 Nov 9;60[1]:678-81. [DOI: 10.1128/AAC.0214015].

34. Wang C, Cai P, Zhan Q, Mi Z, Huang Z, Chen G. Distribution of antiseptic-resistance genes qacA/B in clinical isolates of meticillinresistant Staphylococcus aureus in China. J Hosp Infect. 2008 Aug; 69[4]:393-4. [DOI: 10.1016/j.jhin.2008.05.009].

35. Vali L, Davies SE, Lai LL, Dave J, Amyes SG. Frequency of biocide resistance genes, antibiotic resistance, and the effect of chlorhexidine exposure on clinical methicillin-resistant Staphylococcus aureus isolates. J Antimicrob Chemother. 2008 Mar; 61 [3]: 524-32. [DOI: 10.1093/ jac/dkm520].

36. Alam MM, Kobayashi N, Uehara N, Watanabe N. Analysis on distribution and genomic diversity of high-level antiseptic resistance genes qacA and qacB in human clinical isolates of Staphylococcus aureus. Microb Drug Resist. 2003 Summer; 9 [2]: 109-21. [DOI: 10.1089/ 107662903765826697].

37. Behr H, Reverdy ME, Mabilat C, Freney J, Fleurette J. [Relationship between the level of minimal inhibitory concentrations of five antiseptics and the presence of qacA gene in Staphylococcus aureus]. Pathol Biol [Paris]. 1994 May;42[5]:438-44. French. [PMID: 7824309].

38. Mycock G. Methicillin/antiseptic-resistant Staphylococcus aureus. Lancet. 1985 Oct 26;2 [8461]: 949-50. [DOI: 10.1016/s01406736[85]90881-5].

39. Russell AD, Suller MTE, Maillard JY. Do antiseptics and disinfectants select for antibiotic resistance? J Med Microbiol. $1999 \mathrm{Jul} ; 48[7]: 613$ 615. [DOI: 10.1099/00222615-48-7-613].

40. Leelaporn A, Paulsen IT, Tennent JM, Littlejohn TG, Skurray RA Multidrug resistance to antiseptics and disinfectants in coagulasenegative staphylococci. J Med Microbiol. 1994 Mar; 40 [3]: 214-20. [DOI: 10.1099/00222615-40-3-214]. 

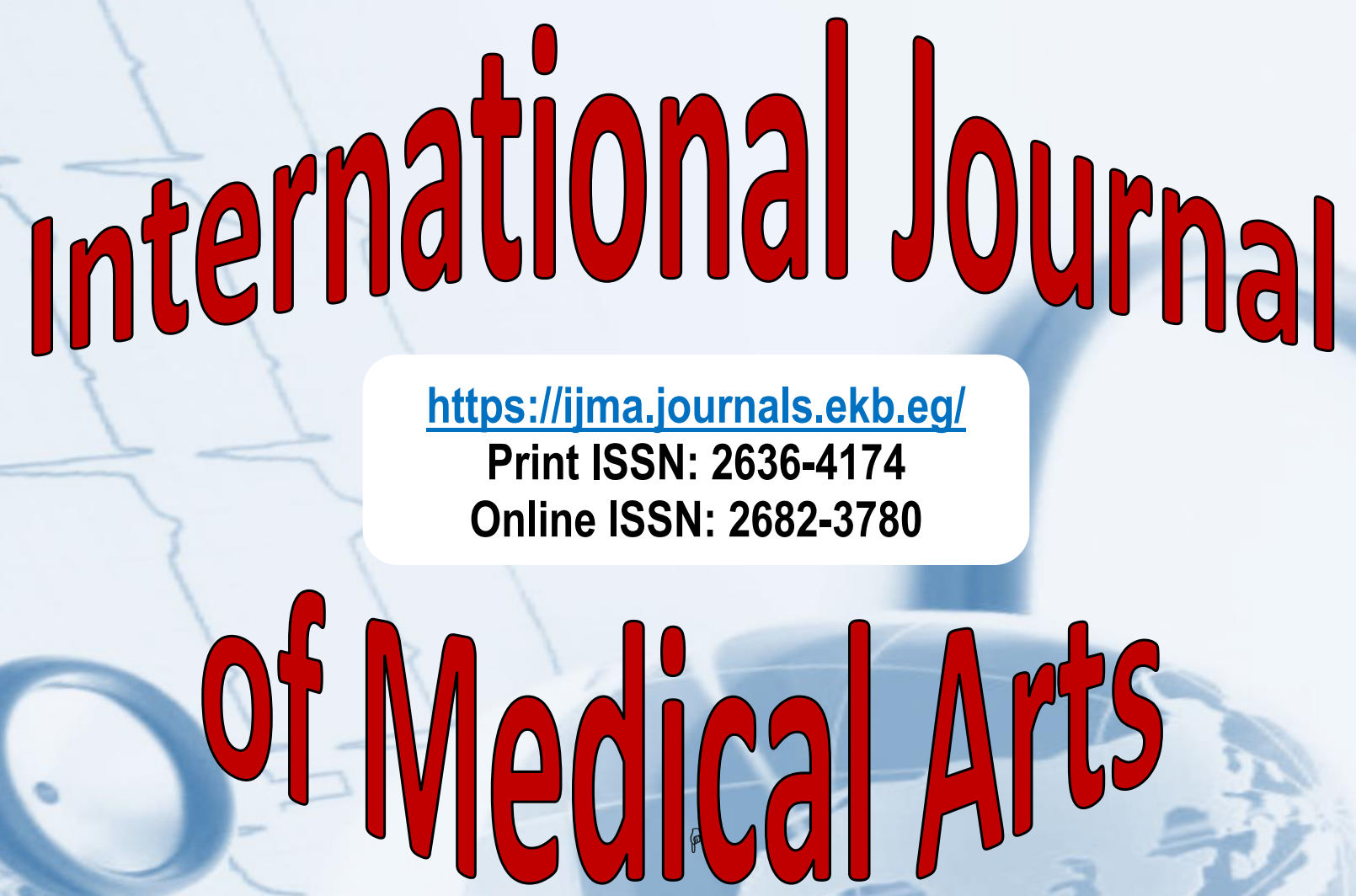